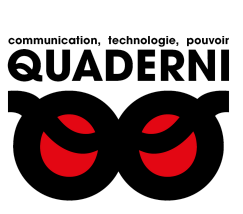

\title{
Quaderni
}

Communication, technologies, pouvoir

\section{Julie Bouchard, Étienne Candel, Hélène Cardy, Gustavo Gomez-Mejia (dir.), La médiatisation de l'évaluation / Evaluation in the media}

\section{Clémentine Gozlan}

\section{(2) OpenEdition \\ 12 Journals \\ Édition électronique \\ URL : https://journals.openedition.org/quaderni/1118 \\ DOI : 10.4000/quaderni. 1118 \\ ISSN : 2105-2956 \\ Éditeur \\ Les éditions de la Maison des sciences de l'Homme}

Édition imprimée

Date de publication : 5 octobre 2017

Pagination : 97-100

Référence électronique

Clémentine Gozlan, « Julie Bouchard, Étienne Candel, Hélène Cardy, Gustavo Gomez-Mejia (dir.), La médiatisation de l'évaluation / Evaluation in the media », Quaderni [En ligne], 94 | Automne 2017, mis en ligne le 05 octobre 2017, consulté le 23 juillet 2021. URL : http://journals.openedition.org/quaderni/ 1118 ; DOI : https://doi.org/10.4000/quaderni.1118 


\section{Compte rendu}

\section{La médiatisation de l'évaluation / \\ Evaluation in the media \\ Julie Bouchard, Étienne Candel, Hélène Cardy, Gustavo Gomez-Mejia (dir.)}

Berne, Peter Lang, 2015

par Clémentine Gozlan

ATER, Université de Versailles

Saint-Quentin-en-Yvelines

1. Michael Power, The Audit Society, New York, Oxford University Press, 1997.

2. Barbara Cassin et al., Derrière les grilles. Sortons du tout-évaluation, Paris, Fayard, 2014.

3. François Vatin (dir.), Évaluer et valoriser. Une sociologie économique de la mesure, Toulouse, Presses Universitaires du Mirail, 2009.
Des ouvrages sur la « société de l'audit $»^{1}$ à ceux qui, dans une perspective critique $^{2}$, contestent l'emprise de l'évaluation sur toutes les sphères de la vie publique et intime, les questions d'évaluation et de mesure ont pris une importance croissante au tournant des années 2000 . C'est à l'exploration de cet objet au cœur de notre actualité que se consacre cet ouvrage collectif, dirigé par Julie Bouchard, Étienne Candel, Hélène Cardy, et Gustavo Gomez-Mejia, tous quatre chercheurs en sciences de l'information et de la communication.

Si les processus d'évaluation et de valorisation ont déjà été étudiés ${ }^{3}$, et qu'ils ne sont par ailleurs pas neufs - Christine Barats rappelle que certains palmarès ont vu le jour dès le $\mathrm{XIX}^{\mathrm{e}}$ siècle - l'ouvrage adopte un angle original : l'espace médiatique a pour particularité d'être à la fois producteur d'instruments d'évaluation, générant, reproduisant et diffusant des valeurs, mais il est aussi lui-même objet d'évaluations, comme le souligne l'introduction stimulante de Julie Bouchard. Évaluation et médias se « coproduisent ». L'ouvrage montre alors en quoi la médiatisation de certains instruments d'évaluation contribue à construire leur puissance et leur légitimation constantes. Les évolutions contemporaines du champ médiatique participent directement à la prospérité et au renouvellement de ces technologies de pouvoir, dont les usages et les acteurs se diversifient considérablement.

À l'appui de cette thèse générale, une variété d'études empiriques est convoquée : les quatorze contributions, dont six en anglais, qui constituent cet ouvrage, sont regroupées en quatre parties : «L'évaluation à l'épreuve 
des médias informatisés », " Des sujets aux institutions, valeurs de l'évaluation », « Les classements et le faire-savoir médiatique », « Les mesures des médias ». Si le lecteur aurait apprécié un chapitre conclusif qui fasse le point sur les apports centraux de l'ouvrage, plusieurs leitmotivs émergent, au terme de la lecture.

Qu'ont de commun l'évaluation artistique à la Nouvelle Star, la fabrique de classements - des universités, des villes, de la liberté de la presse, de l'activité muséale, de blogs... - ; les possibles transformations induites par la mesure du « bonheur »; ou encore la construction d'un baromètre de la « diversité » à la télévision ? Dans la mesure où évaluer, c'est rendre visible un certain point de vue et en occulter un autre, toutes ces opérations d'évaluation reposent sur des présupposés, des représentations de la réalité dont les auteurs grattent la surface pour mieux les dévoiler. Toutes font l'objet d'une médiatisation et d'une diffusion qui, loin d'en stabiliser le sens, rendent ces instruments étonnamment malléables, sujets à des appropriations plurielles, servant des intérêts potentiellement contradictoires. Étudiant la couverture médiatique de Pisa (Programme international de suivi des acquis des élèves) en France, Xavier Pons met en évidence les différentes instrumentalisations politiques de l'étude ; Thierry Baubias souligne que la réception médiatique des palmarès de musées varie selon que l'on considère la presse nationale ou la presse régionale, tandis que les critères sur lesquels repose le palmarès évoluent réciproquement, au gré de l'actualité politique.

Mais ce qui nous semble l'effet le plus spécifique de la médiatisation de l'évaluation, c'est qu'elle engage, enfin, un renouvellement des liens entre pouvoir, autorité et démocratie. Le développement des médias informatisés et des dispositifs qui font appel à la participation citoyenne - «sites d'opinion (tels que les blogs); possibilité laissée aux internautes d'évaluer toutes sortes de choses » (p. 48), ou encore ces talents shows où la définition de la performance revient, in fine, aux spectateurs - transforment l'expertise et redistribuent le pouvoir de juger. C'est alors l'ambiguïté fondamentale de ce tournant que relèvent plusieurs auteurs : ces formes de démocratisation s'exercent au risque de la surveillance généralisée des opinions et des attitudes sociales; au risque, encore, de la reproduction d'une vision néolibérale de la démocratie, dans laquelle l'État, plus que le marché, est perçu comme la plus grande menace envers la liberté de la presse, comme le montrent Francesco Amoretti et Diego Giannone dans leur décryptage des outils de mesure de 
la liberté d'information.

La focale adoptée dans ce recueil rénove ainsi les réflexions sur l'évaluation. Prendre pour objet les liens entre médiatisation et travail évaluatif permet d'une part de dévoiler par quels canaux (par quels « médias ») l'emprise de certains instruments d'évaluation s'effectue. Il s'agit de prendre au sérieux les conditions de la construction et de la diffusion de ces instruments, en observant les acteurs qui les produisent - les médias, certaines ONG... - et ceux qui se les approprient : l'évaluation devient alors un pont entre des univers sociaux pluriels, médiatiques, professionnels, politiques, citoyens.

Il s'agit aussi de montrer que le champ médiatique occupe une place spécifique parmi ces « médias » et est vecteur de formes spécifiques d'évaluation : les contraintes du travail journalistique engagent à la production d'instruments d'évaluation qui s'avèrent les plus aptes à être véhiculés, manipulés et « traduits ». Ces contraintes favorisent, parmi l'éventail des manières de juger, les classements et autres palmarès, qui, faisant « scoop », contribuent à augmenter les ventes des journaux (p. 225). Mais si l'on dit parfois que chiffres et classements parlent d'eux-mêmes, il s'avère au contraire que leur rusticité et leur implacable clarté n'épuisent pas le travail interprétatif, d' « élaboration » (suivant le terme de Wendy Espeland dans sa contribution) des discours, des émotions, et des propositions de réformes. Faciles à politiser, baromètres, indices et autres qualimesures sont tour à tour vecteurs de patrimonialisation, de (re)connaissance, de crédibilité, de contrôle : on peut se demander si la pluralité des usages dont ces instruments peuvent faire l'objet ne contribueraient pas directement à leur circulation et à leur institutionnalisation.

Enfin, si les processus de diffusion et d'imposition de ces pratiques d'évaluation sont minutieusement décrits, le processus symétrique n'est que marginalement traité dans cet ouvrage. D'une certaine manière, les cas choisis pour étudier la médiatisation de l'évaluation sont tous des cas de « succès ». Pourtant, certaines contributions soulignent bien que ces instruments ne sont pas tous performatifs de la même manière, qu'ils peuvent être remplacés par des instruments alternatifs dans certains mondes sociaux, voire rejetés par une partie des acteurs - comme le Musée d'Orsay, qui a refusé de participer au palmarès des musées de France. L'étude des mouvements sociaux qui contestent ces dispositifs, et qui se construisent autour de sujets sensibles 
abordés dans l'ouvrage, comme la marchandisation des données personnelles, pourrait offrir des contrepoints intéressants. Plus que des critiques, ces quelques pistes invitent à prolonger les réflexions et les perspectives d'analyse engageantes ouvertes par cet ouvrage. 